\title{
Asthma, bronchitis respiratory symptoms, allergies and home environment: how are they related?
}

\author{
Agata Wypych-Ślusarska, Ewa Niewiadomska, Joanna Głogowska-Ligus \\ Department of Epidemiology and Biostatistics, School of Health Sciences in Bytom, Medical University of Silesia in Katowice, Poland \\ Adv Dermatol Allergol 2022; XXXIX (4): 729-738 \\ DOI: https://doi.org/10.5114/ada.2021.109696
}

\begin{abstract}
Introduction: Home environmental conditions can affect the prevalence of childhood asthma and allergies. Aim: To assess the relationship between the prevalence of childhood asthma, bronchitis, and allergies and the condition of the home environment.

Material and methods: In 2018 and 2019, a cross-sectional study on 2932 children from elementary schools in the Silesian Voivodship (Southern Poland) was conducted. The questionnaire was based on the International Study of Asthma and Allergies in Childhood (ISAAC). In order to determine the association between the home environment (presence of moulds, furry pets) and respiratory symptoms and diseases, a logistic regression analysis was performed by calculating the odds ratio $(\mathrm{OR})$, determining $p<0.05$ as the level of significance.

Results: Asthma risk factors were male sex, heating with solid fuel and presence of moulds. The prevalence of respiratory symptoms, bronchitis, and allergic diseases is statistically more common with the presence of moulds in dwellings. A protective effect of the presence of pets on the prevalence of asthma (OR $=0.77 ; 95 \% \mathrm{Cl}: 0.59-0.99)$, allergy to pet allergens $(\mathrm{OR}=0.59 ; 95 \% \mathrm{Cl}: 0.45-0.76)$, allergy to house dust mite $(\mathrm{OR}=0.70 ; 95 \% \mathrm{Cl}: 0.56-0.87)$ and wheeze in the last 12 months $(\mathrm{OR}=0.70 ; 95 \% \mathrm{Cl}: 0.54-0.91)$ and ever $(\mathrm{OR}=0.85 ; 95 \% \mathrm{Cl}$ : 0.70-1.02) was observed. Conclusions: The study confirmed the known adverse influence of the presence of moulds and heating with solid fuel on the prevalence of asthma, bronchitis, respiratory symptoms and allergic diseases. The protective influence of pets on the occurrence of the health disorders under study was demonstrated.
\end{abstract}

Key words: home environment, asthma, allergy, mould, children.

\section{Introduction}

Asthma and allergies are among the most common chronic childhood diseases and represent a significant public health problem. Epidemiological data show a rising prevalence of these diseases, mainly in highly developed countries [1]. The International Study of Asthma and Allergy in Childhood (ISAAC) is one of the few studies demonstrating the epidemiology of childhood asthma on a global basis [2]. ISAAC Phase Three highlighted the increasing prevalence of that disease and its global diversity [2]. Globally, asthma affects $11.7 \%$ of children aged 6-7 years and $14.1 \%$ of children aged $13-14$ years, allergic rhinitis and conjunctivitis, $8.5 \%$ and $14.6 \%$, respectively, and eczema $-7.9 \%$ and $7.3 \%$, respectively [2]. Up-to-date results on the prevalence of childhood asthma and allergic diseases are obtained from studies conducted in smaller centres and consequently they show a regional situation in that regard. A nationwide study called Epidemiology of Allergic Diseases in Poland (ECAP) revealed that features of allergy are present in $40 \%$ of children aged $6-7$ years and $43 \%$ of children aged $13-14$ years, whereas asthma affects $4.4 \%$ and $6.5 \%$, respectively [3]. A study conducted among schoolchildren in Bytom aged 13-16 years showed the prevalence of bronchial asthma of $9.7 \%$ [4]. The increasing prevalence of asthma and allergic diseases may be partly explained by better diagnostic possibilities, growing parental awareness of the symptoms of those disorders as well as the impact of environmental factors, with the home environment being of considerable significance. Studies also point to a possible relationship between environmental tobacco smoke (ETS), the presence of mould and dampness in the dwelling, pet allergens, and the occurrence of allergic diseases

Address for correspondence: Agata Wypych-Ślusarska PhD, Department of Epidemiology and Biostatistics, School of Health Sciences, Medical University of Silesia, 18 Piekarska St, 41-902 Bytom, Poland, phone: 501239 881, e-mail: awypych@sum.edu.pl Received: 30.12 .2020 , accepted: 10.06 .2021$. 
and asthma [5-8]. The direction of that relationship is not always clear for each factor. For instance, there is some debate as to whether the presence of pets is a risk factor or a protective factor for the above health disorders. Moreover, the hygiene hypothesis of asthma and allergy and its modified version - the gut microbial deprivation hypothesis - indicate that the excessive sanitization of the environment leads to a reduction in the number of infections and changes the intestinal colonization by reducing exposure to microorganisms, thereby disrupting the development of the immune system. Consequently, it becomes a predisposing factor for the development of allergies and asthma [9-11].

\section{Aim}

Given the above, a study was conducted to assess the relationship between the factors of the home environment and the prevalence of asthma, bronchitis, respiratory symptoms, and allergic diseases among children.

\section{Material and methods}

\section{Study design}

In 2018 and 2019, an epidemiological cross-sectional study on 2932 children from elementary schools in the Silesian Voivodship (Southern Poland) was conducted. The study covered children from selected counties (powiat) of the Silesian Voivodship chosen due to their location in the particular subregions of the Silesian Voivodship (northern, central, western, and southern). Data on the health and socio-economic situation of the children's families were collected using a questionnaire distributed in primary schools. Four thousand nine hundred and twenty-eight parents were invited to the study, and answers from 2932 participants were obtained (response rate $59.5 \%)$. Cluster sampling was used to select places of residence from the chosen counties of the Silesian Voivodship and then schools from the given places of residence/areas. The study inclusion criterion was consent from the school principal to carry out the study. The data on the number of inhabitants of the county by age and sex obtained from Statistics Poland made it possible to calculate the minimum sample size for each county of the Silesian Voivodship [12]. The total number of children aged 6 to 15 in the separate counties of Silesian Voivodeship was taken into account as the size of the population. $50 \%$ sample proportion, 95\% confidence level, and 5\% margin of error were taken to determine the appropriate sample size for estimating the proportion of population. As a result we obtained a total number of 2239 children aged between 6 and 15 years. In total, data on 2932 children were collected, including 1432 (48.8\%) boys and 1500 (51.2\%) girls. The average age was $10.7 \pm 2.2$ years. Due to the highly urbanized territorial structure of the Silesian Voivodship, the vast majority of the respondents lived in towns (91.7\%), the remaining schoolchildren were rural residents.

\section{Questionnaire and statistical analyses}

The children's parents completed a questionnaire based on the International Study of Asthma and Allergies in Childhood. The home environment is defined by the following factors: the presence of moulds (yes/no), furry pets (present/absent), exposure to environmental tobacco smoke (present/absent), and type of heating (heating with solid fuel/central heating). Exposure to ETS was defined on the basis of affirmative responses to the questionnaire indicating that the child's mother and/or father and/or another person living in the same dwelling smoked tobacco. Heating with solid fuel was defined as heating using coal, wood, pellet, or eco-pea coal. Chronic cough meant cough that lasted at least 3 months. The children were divided into different age groups corresponding to the particular grades of primary school: 6-10 years of age (grades 1-3) $50.5 \%, 11-12$ years of age (grades $4-6$ ) $-25.3 \%$ and $13-15$ years of age (grades $7-8$ ) $-24.2 \%$. The primary classification was the age of children. The age distribution of the groups adopted in the ISAAC study methodology (6-7 and 13-14 years of age) was not possible in that case due to a small number of children in the 6-7 years age group. Therefore, we used a wide age range corresponding to the grade of the analysed groups.

\section{Statistical analysis}

The $\chi^{2}$ test was used to assess the relationship between the qualitative variables, determining $p<0.05$ as the level of significance. The influence of demographic and environmental factors on the occurrence of asthma, bronchitis, respiratory symptoms, and allergic diseases among children was verified by univariate logistic regression. Unadjusted models (crude odds ratios) with one independent variable of: gender, age groups, heating with solid fuel, presence of mould or dampness in dwelling, exposure to tobacco smoke, presence of pets in dwelling, were used.

The statistical analyses were performed using the procedures available in Statistica 13.0.

\section{Results}

The prevalence of bronchial asthma declared in the questionnaire and diagnosed ever was $9.3 \%$, and of obstructive bronchitis - 16.6\%. Chronic cough affected 9.8\% of the children, attacks of dyspnoea ever and in the last 12 months: $11.2 \%$ and $4.9 \%$, respectively, wheezing ever and in the last 12 months: $21.5 \%$ and $9.6 \%$, respectively. Allergy to pollen affected $21.1 \%$ of the children, to house dust mites $-14.5 \%$, and to pet allergens $-9.1 \%$.

Tables 1 and 2 show the relationship between the occurrence of respiratory symptoms, allergic diseases, 
Table 1. Prevalence of respiratory symptoms broken down by sex and age

\begin{tabular}{|c|c|c|c|c|}
\hline \multirow{2}{*}{$\begin{array}{l}\text { Health problem } \\
\text { Chronic cough }\end{array}$} & \multicolumn{2}{|c|}{ Variable } & \multirow{2}{*}{$\frac{n(\%)}{150(10.5)}$} & \multirow{2}{*}{$\frac{P \text {-value }}{\text { A }^{\mathrm{A}}}$} \\
\hline & Sex & Male & & \\
\hline & & Female & $137(9.1)$ & \\
\hline & Age & $6-10$ & $169(11.5)$ & 0.008 \\
\hline & & $11-12$ & $85(7.9)$ & \\
\hline & & $13-15$ & $33(8.7)$ & \\
\hline \multirow[t]{5}{*}{ Dyspnoea ever } & Sex & Male & 187 (13.1) & 0.001 \\
\hline & & Female & $140(9.3)$ & \\
\hline & Age & $6-10$ & $182(12.4)$ & 0.08 \\
\hline & & $11-12$ & $103(9.6)$ & \\
\hline & & $13-15$ & 42 (11.0) & \\
\hline \multirow{5}{*}{$\begin{array}{l}\text { Dyspnoea in } \\
\text { the last } 12 \\
\text { months }\end{array}$} & Sex & Male & $85(5.9)$ & 0.01 \\
\hline & & Female & $58(3.9)$ & \\
\hline & Age & $6-10$ & $79(5.4)$ & 0.4 \\
\hline & & $11-12$ & $48(4.5)$ & \\
\hline & & $13-15$ & $16(4.2)$ & \\
\hline \multirow[t]{5}{*}{ Wheeze ever } & Sex & Male & $347(24.2)$ & $<0.001$ \\
\hline & & Female & $282(18.8)$ & \\
\hline & Age & $6-10$ & $353(24.0)$ & 0.003 \\
\hline & & $11-12$ & 207 (19.2) & \\
\hline & & $13-15$ & 69 (18.1) & \\
\hline \multirow{5}{*}{$\begin{array}{l}\text { Wheeze in the } \\
\text { last } 12 \text { months }\end{array}$} & Sex & Male & $156(10.9)$ & 0.02 \\
\hline & & Female & $126(8.4)$ & \\
\hline & Age & $6-10$ & $157(10.7)$ & 0.1 \\
\hline & & $11-12$ & $95(8.8)$ & \\
\hline & & $13-15$ & $30(7.9)$ & \\
\hline
\end{tabular}

the $\chi^{2}$ test.

asthma, and bronchitis under investigation, depending on the sex and age of the child.

Chronic cough, wheeze ever, and bronchitis were statistically significantly more common in younger children, whereas allergy to pollen was statistically significantly more common in the oldest children. Wheeze, dyspnoea, asthma, bronchitis, and all allergic diseases under study affected boys more frequently than girls, with the differences between the groups being statistically significant.

The relationship between the home environment and the prevalence of respiratory symptoms, asthma, bronchitis, and allergic diseases is shown in Tables 3 and 4.

Heating with solid fuel affected the prevalence of wheeze in the last 12 months, asthma, and allergy to house dust mites in a statistically significant manner. The respiratory symptoms, allergic diseases, asthma, and bronchitis under study were statistically significantly more common in children occupying dwellings with traces of
Table 2. Prevalence of allergic diseases, asthma and bronchitis broken down by sex and age

\begin{tabular}{|c|c|c|c|c|}
\hline Health problem & \multicolumn{2}{|c|}{ Variable } & $n(\%)$ & $P$-value ${ }^{A}$ \\
\hline \multirow[t]{5}{*}{ Asthma } & \multirow[t]{2}{*}{ Sex } & Male & $163(11.4)$ & \multirow[t]{2}{*}{$<0.001$} \\
\hline & & Female & $110(7.3)$ & \\
\hline & \multirow[t]{3}{*}{ Age } & $6-10$ & 149 (10.1) & \multirow[t]{3}{*}{0.1} \\
\hline & & $11-12$ & $85(7.9)$ & \\
\hline & & $13-15$ & 39 (10.2) & \\
\hline \multirow[t]{5}{*}{ Bronchitis } & \multirow[t]{2}{*}{ Sex } & Male & $226(18.7)$ & \multirow[t]{2}{*}{0.004} \\
\hline & & Female & $221(14.7)$ & \\
\hline & \multirow[t]{3}{*}{ Age } & $6-10$ & $288(19.6)$ & \multirow[t]{3}{*}{$<0.001$} \\
\hline & & $11-12$ & $146(13.5)$ & \\
\hline & & $13-15$ & $44(14.2)$ & \\
\hline \multirow{5}{*}{$\begin{array}{l}\text { Allergy to house } \\
\text { dust mite }\end{array}$} & \multirow[t]{2}{*}{ Sex } & Male & $249(17.4)$ & \multirow[t]{2}{*}{$<0.001$} \\
\hline & & Female & $177(11.8)$ & \\
\hline & \multirow[t]{3}{*}{ Age } & $6-10$ & $208(14.1)$ & \multirow[t]{3}{*}{0.09} \\
\hline & & $11-12$ & 149 (13.8) & \\
\hline & & $13-15$ & 69 (18.2) & \\
\hline \multirow[t]{5}{*}{ Allergy to pollen } & \multirow[t]{2}{*}{ Sex } & Male & $344(24.1)$ & \multirow[t]{2}{*}{$<0.001$} \\
\hline & & Female & $274(18.3)$ & \\
\hline & \multirow[t]{3}{*}{ Age } & $6-10$ & $285(13.4)$ & \multirow[t]{3}{*}{0.04} \\
\hline & & $11-12$ & $240(22.3)$ & \\
\hline & & $13-15$ & $93(24.5)$ & \\
\hline \multirow[t]{5}{*}{ Pet allergy } & \multirow[t]{2}{*}{ Sex } & Male & $150(10.5)$ & \multirow[t]{2}{*}{0.01} \\
\hline & & Female & $116(7.7)$ & \\
\hline & \multirow[t]{3}{*}{ Age } & $6-10$ & $138(9.4)$ & \multirow[t]{3}{*}{0.8} \\
\hline & & $11-12$ & $94(8.7)$ & \\
\hline & & $13-15$ & $34(9.0)$ & \\
\hline
\end{tabular}

the $\chi^{2}$ test.

mould or damp. Chronic cough and dyspnoea in the last 12 months more frequently affected children exposed to ETS. A protective influence of the presence of pets on wheeze ever and in the last 12 months, asthma and allergy to house dust and pet allergens was observed.

A regression analysis (Tables 5,6 ) confirmed that the risk of asthma was significantly higher in male children $(\mathrm{OR}=1.54 ; 95 \% \mathrm{Cl}: 1.19-1.99)$. Central heating proved to be a protective factor in asthma $(\mathrm{OR}=0.67 ; 95 \% \mathrm{Cl}$ : 0.49-0.93) and wheeze in the last 12 months $(\mathrm{OR}=0.68$; 95\% Cl: 0.49-0.94). The male sex increased the risk of bronchitis (OR $=1.28 ; 95 \% \mathrm{Cl}: 1.05-1.57)$, dyspnoea ever $(\mathrm{OR}=1.39 ; 95 \% \mathrm{Cl}: 1.09-1.76)$ and in the last 12 months $(O R=1.44 ; 95 \% \mathrm{Cl}: 1.01-2.04)$, as well as wheeze ever $(\mathrm{OR}=1.32 ; 95 \% \mathrm{Cl}: 1.10-1.58)$. The allergic diseases under study affected boys rather than girls significantly more often (Table 6). The children from the 11-12 years age group suffered significantly less often from chronic 
Table 3. Home environment and respiratory symptoms

\begin{tabular}{|c|c|c|c|c|}
\hline $\begin{array}{l}\text { Health } \\
\text { problem }\end{array}$ & \multicolumn{2}{|c|}{ Home environment } & $n(\%)$ & $P$-value ${ }^{\mathrm{A}}$ \\
\hline \multirow[t]{8}{*}{$\begin{array}{l}\text { Chronic } \\
\text { cough }\end{array}$} & \multirow[t]{2}{*}{$\begin{array}{l}\text { Type of } \\
\text { heating }\end{array}$} & $\begin{array}{l}\text { Central } \\
\text { heating }\end{array}$ & $236(9.5)$ & \multirow[t]{2}{*}{0.3} \\
\hline & & $\begin{array}{l}\text { Heating with } \\
\text { solid fuel }\end{array}$ & 47 (11.0) & \\
\hline & \multirow{2}{*}{$\begin{array}{l}\text { Moulds/ } \\
\text { dampness }\end{array}$} & Present & $101(14.4)$ & \multirow[t]{2}{*}{$<0.001$} \\
\hline & & Absent & $183(8.3)$ & \\
\hline & \multirow[t]{2}{*}{ Pet allergens } & Present & $175(9.6)$ & \multirow[t]{2}{*}{0.7} \\
\hline & & Absent & $111(10.1)$ & \\
\hline & \multirow{2}{*}{$\begin{array}{l}\text { Environmental } \\
\text { tobacco smoke }\end{array}$} & Present & $129(11.7)$ & \multirow[t]{2}{*}{0.004} \\
\hline & & Absent & $152(8.5)$ & \\
\hline \multirow[t]{8}{*}{$\begin{array}{l}\text { Dyspnoea } \\
\text { ever }\end{array}$} & \multirow[t]{2}{*}{$\begin{array}{l}\text { Type of } \\
\text { heating }\end{array}$} & $\begin{array}{l}\text { Central } \\
\text { heating }\end{array}$ & $\begin{array}{c}272 \\
(10.9)\end{array}$ & \multirow[t]{2}{*}{0.6} \\
\hline & & $\begin{array}{c}\text { Heating } \\
\text { with solid } \\
\text { fuel }\end{array}$ & 51 (11.9) & \\
\hline & \multirow{2}{*}{$\begin{array}{c}\text { Moulds/ } \\
\text { dampness }\end{array}$} & Present & 99 (14.1) & \multirow[t]{2}{*}{$<0.001$} \\
\hline & & Absent & $227(10.2)$ & \\
\hline & \multirow[t]{2}{*}{ Pet allergens } & Present & $196(10.8)$ & \multirow[t]{2}{*}{0.3} \\
\hline & & Absent & $131(11.9)$ & \\
\hline & \multirow{2}{*}{$\begin{array}{l}\text { Environmental } \\
\text { tobacco smoke }\end{array}$} & Present & 132 (11.9) & \multirow[t]{2}{*}{0.3} \\
\hline & & Absent & $191(10.6)$ & \\
\hline \multirow{6}{*}{$\begin{array}{l}\text { Dyspnoea } \\
\text { in the } \\
\text { last } \\
12 \text { months }\end{array}$} & \multirow[t]{2}{*}{$\begin{array}{l}\text { Type of } \\
\text { heating }\end{array}$} & $\begin{array}{l}\text { Central } \\
\text { heating }\end{array}$ & $114(4.6)$ & \multirow[t]{2}{*}{0.2} \\
\hline & & $\begin{array}{l}\text { Heating } \\
\text { with solid } \\
\text { fuel }\end{array}$ & $26(6.1)$ & \\
\hline & \multirow{2}{*}{$\begin{array}{l}\text { Moulds/ } \\
\text { dampness }\end{array}$} & Present & $44(6.3)$ & \multirow[t]{2}{*}{0.005} \\
\hline & & Absent & $98(4.4)$ & \\
\hline & \multirow[t]{2}{*}{ Pet allergens } & Present & $83(4.6)$ & \multirow[t]{2}{*}{0.3} \\
\hline & & Absent & $60(5.5)$ & \\
\hline
\end{tabular}

cough $(\mathrm{OR}=0.66 ; 95 \% \mathrm{Cl}: 0.49-0.91)$, wheeze ever (OR $=0.74 ; 95 \% \mathrm{Cl}: 0.60-0.93)$, dyspnoea ever $(\mathrm{OR}=0.68$; $95 \% \mathrm{Cl}: 0.51-0.92)$ and bronchitis (OR $=0.61 ; 95 \% \mathrm{Cl}$ : $0.48-0.79)$ than children from the $6-10$ years age group. Moreover, the regression analysis showed that the occurrence of respiratory symptoms (apart from dyspnoea in the last 12 months), bronchitis, and analysed allergic diseases became statistically more common with the presence of moulds in children's dwellings. ETS is a factor significantly affecting chronic cough $(\mathrm{OR}=1.35$; 95\% Cl: 1.43-2.45). Furthermore, the obtained results suggest a protective effect of the presence of pets on the prevalence of asthma (OR $=0.77 ; 95 \% \mathrm{Cl}$ : 0.59-0.99), pet allergy $(\mathrm{OR}=0.59 ; 95 \% \mathrm{Cl}: 0.45-0.76)$, allergy to house dust mite $(\mathrm{OR}=0.70 ; 95 \% \mathrm{Cl}: 0.56-0.87)$ and wheeze in the last 12 months (OR $=0.70 ; 95 \% \mathrm{Cl}$ : $0.54-0.91)$.

\begin{tabular}{|c|c|c|c|c|}
\hline \multirow{3}{*}{$\begin{array}{l}\text { Health } \\
\text { problem }\end{array}$} & \multicolumn{2}{|c|}{ Home environment } & \multirow{2}{*}{$\begin{array}{c}n(\%) \\
66(6.0)\end{array}$} & \multirow{2}{*}{$\begin{array}{c}P \text {-value } \\
0.03\end{array}$} \\
\hline & Environmental & Present & & \\
\hline & tobacco smoke & Absent & $75(4.2)$ & \\
\hline \multirow[t]{8}{*}{$\begin{array}{l}\text { Wheeze } \\
\text { ever }\end{array}$} & \multirow[t]{2}{*}{$\begin{array}{l}\text { Type of } \\
\text { heating }\end{array}$} & $\begin{array}{l}\text { Central } \\
\text { heating }\end{array}$ & $\begin{array}{c}519 \\
(20.9) \\
\end{array}$ & 0.2 \\
\hline & & $\begin{array}{l}\text { Heating } \\
\text { with solid } \\
\text { fuel }\end{array}$ & $\begin{array}{c}101 \\
(23.6)\end{array}$ & \\
\hline & \multirow[t]{2}{*}{$\begin{array}{l}\text { Moulds/ } \\
\text { dampness }\end{array}$} & Present & $\begin{array}{c}183 \\
(26.0)\end{array}$ & $<0.001$ \\
\hline & & Absent & $\begin{array}{c}443 \\
(20.0)\end{array}$ & \\
\hline & \multirow[t]{2}{*}{ Pet allergens } & Present & $\begin{array}{c}368 \\
(20.3) \\
\end{array}$ & 0.03 \\
\hline & & Absent & $\begin{array}{c}260 \\
(23.6)\end{array}$ & \\
\hline & \multirow[t]{2}{*}{$\begin{array}{l}\text { Environmental } \\
\text { tobacco smoke }\end{array}$} & Present & $\begin{array}{l}248 \\
(22.4)\end{array}$ & 0.3 \\
\hline & & Absent & $\begin{array}{c}375 \\
(20.9)\end{array}$ & \\
\hline \multirow{8}{*}{$\begin{array}{l}\text { Wheeze } \\
\text { in the } \\
\text { last } \\
12 \text { months }\end{array}$} & \multirow[t]{2}{*}{$\begin{array}{l}\text { Type of } \\
\text { heating }\end{array}$} & $\begin{array}{l}\text { Central } \\
\text { heating }\end{array}$ & $219(8.8)$ & 0.005 \\
\hline & & $\begin{array}{l}\text { Heating } \\
\text { with solid } \\
\text { fuel }\end{array}$ & $56(13.1)$ & \\
\hline & \multirow{2}{*}{$\begin{array}{l}\text { Moulds/ } \\
\text { dampness }\end{array}$} & Present & $92(13.1)$ & $<0.001$ \\
\hline & & Absent & $188(8.5)$ & \\
\hline & \multirow[t]{2}{*}{ Pet allergens } & Present & $152(8.4)$ & 0.003 \\
\hline & & Absent & $129(11.7)$ & \\
\hline & \multirow{2}{*}{$\begin{array}{l}\text { Environmental } \\
\text { tobacco smoke }\end{array}$} & Present & $110(9.9)$ & 0.6 \\
\hline & & Absent & $168(9.4)$ & \\
\hline
\end{tabular}

athe $\chi^{2}$ test.

\section{Discussion}

The subject matter and scope of the planned study made it possible to obtain information on the importance of the factors of the home environment in relation to the prevalence of respiratory symptoms, allergic diseases, asthma, and bronchitis. The choice of the subject matter was dictated by the seriousness of the problem: on the one hand, the rising prevalence of asthma, allergic diseases, and respiratory symptoms and, on the other hand, the necessity to assess the influence of home environment factors on the prevalence of those health disorders. Although a large number of epidemiological studies have explored that problem, no clear consensus exists over the direction of the influence in relation to the particular factors. 
Table 4. Home environment and allergic diseases, asthma and bronchitis

\begin{tabular}{|c|c|c|c|c|}
\hline \multirow{2}{*}{$\begin{array}{l}\text { Health problem } \\
\text { Asthma }\end{array}$} & \multicolumn{2}{|c|}{ Home environment } & \multirow{2}{*}{$\begin{array}{c}n(\%) \\
218(8.8)\end{array}$} & \multirow{2}{*}{$\frac{P \text {-value }}{A}$} \\
\hline & \multirow[t]{2}{*}{ Type of heating } & Central heating & & \\
\hline & & Heating with solid fuel & $53(12.4)$ & \\
\hline & \multirow[t]{2}{*}{ Moulds/dampness } & Present & $84(12.0)$ & 0.006 \\
\hline & & Absent & $188(8.5)$ & \\
\hline & \multirow[t]{2}{*}{ Pet allergens } & Present & $153(8.4)$ & 0.03 \\
\hline & & Absent & $119(10.8)$ & \\
\hline & \multirow[t]{2}{*}{ Environmental tobacco smoke } & Present & $114(10.3)$ & 0.1 \\
\hline & & Absent & $155(8.6)$ & \\
\hline \multirow[t]{8}{*}{ Bronchitis } & \multirow[t]{2}{*}{ Type of heating } & Central heating & $402(16.2)$ & 0.1 \\
\hline & & Heating with solid fuel & $81(19.0)$ & \\
\hline & \multirow[t]{2}{*}{ Moulds/dampness } & Present & $145(20.6)$ & 0.001 \\
\hline & & Absent & $341(15.1)$ & \\
\hline & \multirow[t]{2}{*}{ Pet allergens } & Present & $287(15.8)$ & 0.1 \\
\hline & & Absent & 199 (18.1) & \\
\hline & \multirow[t]{2}{*}{ Environmental tobacco smoke } & Present & 201 (18.2) & 0.07 \\
\hline & & Absent & $280(15.6)$ & \\
\hline \multirow{8}{*}{$\begin{array}{l}\text { Allergy to house } \\
\text { dust mite }\end{array}$} & \multirow[t]{2}{*}{ Type of heating } & Central heating & 346 (13.9) & 0.03 \\
\hline & & Heating with solid fuel & 77 (18.0) & \\
\hline & \multirow[t]{2}{*}{ Moulds/dampness } & Present & $140(19.9)$ & $<0.001$ \\
\hline & & Absent & $283(12.8)$ & \\
\hline & \multirow[t]{2}{*}{ Pet allergens } & Present & $233(12.8)$ & $<0.001$ \\
\hline & & Absent & $191(17.1)$ & \\
\hline & \multirow[t]{2}{*}{ Environmental tobacco smoke } & Present & $160(14.5)$ & 1.0 \\
\hline & & Absent & $260(14.5)$ & \\
\hline \multirow[t]{8}{*}{ Allergy to pollen } & \multirow[t]{2}{*}{ Type of heating } & Central heating & $532(21.4)$ & 0.4 \\
\hline & & Heating with solid fuel & $83(19.4)$ & \\
\hline & \multirow[t]{2}{*}{ Moulds/dampness } & Present & $167(23.8)$ & 0.04 \\
\hline & & Absent & $449(20.3)$ & \\
\hline & \multirow[t]{2}{*}{ Pet allergens } & Present & $369(20.3)$ & 0.2 \\
\hline & & Absent & $246(22.4)$ & \\
\hline & \multirow[t]{2}{*}{ Environmental tobacco smoke } & Present & $221(20.0)$ & 0.3 \\
\hline & & Absent & $388(21.6)$ & \\
\hline \multirow[t]{8}{*}{ Pet allergy } & \multirow[t]{2}{*}{ Type of heating } & Central heating & $220(8.9)$ & 0.3 \\
\hline & & Heating with solid fuel & $44(10.3)$ & \\
\hline & \multirow[t]{2}{*}{ Moulds/dampness } & Present & $81(11.5)$ & 0.008 \\
\hline & & Absent & $183(8.3)$ & \\
\hline & Pet allergens & Present & $134(7.4)$ & $<0.001$ \\
\hline & & Absent & $130(11.9)$ & \\
\hline & Environmental tobacco smoke & Present & $102(9.3)$ & 0.8 \\
\hline & & Absent & $160(8.9)$ & \\
\hline
\end{tabular}

Athe $\chi^{2}$ test. 
Table 5. Crude odds ratios (OR) and $95 \%$ confidence intervals related to determinants of observed respiratory symptoms

\begin{tabular}{|c|c|c|c|c|c|}
\hline $\begin{array}{l}\text { Health } \\
\text { problem }\end{array}$ & $\begin{array}{l}\text { Determinants } \\
\text { *reference group }\end{array}$ & $\begin{array}{l}\text { Regression } \\
\text { coefficient }\end{array}$ & $\begin{array}{l}P \text {-value of the } \\
\text { regression coefficient }\end{array}$ & OR & $95 \% \mathrm{Cl}$ \\
\hline \multirow{8}{*}{$\begin{array}{l}\text { Chronic } \\
\text { cough }\end{array}$} & $\operatorname{Sex}\left(F^{\star} / M\right)$ & 0.12 & 0.3 & 1.13 & $0.88-1.45$ \\
\hline & Age groups: & & & & \\
\hline & $11-12^{*} / 13-15$ & 0.008 & 0.9 & 1.00 & $0.61-1.66$ \\
\hline & $6-10^{\star} / 11-12$ & -0.4 & 0.01 & 0.66 & $0.49-0.91$ \\
\hline & $\begin{array}{l}\text { Type of heating (heating with solid fuel*/ } \\
\text { central heating) }\end{array}$ & -0.01 & 0.9 & 0.98 & $0.64-1.49$ \\
\hline & Moulds/dampness $\left(\mathrm{N}^{*} / \mathrm{Y}\right)$ & 0.62 & $<0.001$ & 1.87 & $1.43-2.45$ \\
\hline & ETS $\left(N^{*} / Y\right)$ & 0.30 & 0.01 & 1.35 & $1.05-1.74$ \\
\hline & Pet allergens $\left(N^{*} / Y\right)$ & -0.03 & 0.7 & 0.96 & $0.74-1.24$ \\
\hline \multirow{8}{*}{$\begin{array}{l}\text { Dyspnoea } \\
\text { ever }\end{array}$} & $\operatorname{Sex}\left(F^{\star} / M\right)$ & 0.32 & 0.006 & 1.39 & $1.09-1.76$ \\
\hline & Age groups: & & & & \\
\hline & $11-12^{*} / 13-15$ & 0.25 & 0.1 & 1.28 & $0.91-1.82$ \\
\hline & $6-10^{*} / 11-12$ & -0.38 & 0.01 & 0.68 & $0.51-0.92$ \\
\hline & $\begin{array}{l}\text { Type of heating (heating with solid fuel*/ } \\
\text { central heating) }\end{array}$ & -0.01 & 0.8 & 0.98 & $0.73-1.30$ \\
\hline & Moulds/dampness $\left(\mathrm{N}^{\star} / \mathrm{Y}\right)$ & 0.36 & 0.006 & 1.43 & $1.10-1.86$ \\
\hline & $\operatorname{ETS}\left(N^{*} / Y\right)$ & 0.11 & 0.3 & 1.11 & $0.87-1.42$ \\
\hline & Pet allergens $\left(\mathrm{N}^{*} / \mathrm{Y}\right)$ & -0.07 & 0.5 & 0.92 & $0.72-1.18$ \\
\hline \multirow{8}{*}{$\begin{array}{l}\text { Dyspnoea in } \\
\text { the last } \\
12 \text { months }\end{array}$} & $\operatorname{Sex}\left(F^{*} / M\right)$ & 0.36 & 0.03 & 1.44 & $1.01-2.04$ \\
\hline & Age groups: & & & & \\
\hline & $11-12^{*} / 13-15$ & 0.05 & 0.8 & 1.06 & $0.63-1.76$ \\
\hline & $6-10^{*} / 11-12$ & -0.18 & 0.3 & 0.82 & $0.55-1.25$ \\
\hline & $\begin{array}{l}\text { Type of heating (heating with solid fuel }{ }^{*} / \\
\text { central heating) }\end{array}$ & -0.20 & 0.3 & 0.81 & $0.52-1.28$ \\
\hline & Moulds/dampness $\left(\mathrm{N}^{*} / \mathrm{Y}\right)$ & 0.33 & 0.08 & 1.39 & $0.95-2.03$ \\
\hline & $\operatorname{ETS}\left(N^{*} / \mathrm{Y}\right)$ & 0.34 & 0.05 & 1.40 & $0.99-1.99$ \\
\hline & Pet allergens $\left(N^{*} / \mathrm{Y}\right)$ & -0.20 & 0.2 & 0.81 & $0.57-1.15$ \\
\hline \multirow[t]{8}{*}{ Wheeze ever } & $\operatorname{Sex}\left(F^{\star} / M\right)$ & 0.28 & 0.002 & 1.32 & $1.10-1.58$ \\
\hline & Age groups: & & & & \\
\hline & $11-12^{*} / 13-15$ & -0.02 & 0.9 & 0.97 & $0.72-1.31$ \\
\hline & $6-10^{*} / 11-12$ & -0.29 & 0.009 & 0.74 & $0.60-0.93$ \\
\hline & $\begin{array}{l}\text { Type of heating (heating with solid fuel }{ }^{\star} / \\
\text { central heating) }\end{array}$ & -0.09 & 0.4 & 0.90 & $0.70-1.16$ \\
\hline & Moulds/dampness $\left(\mathrm{N}^{*} / \mathrm{Y}\right)$ & 0.33 & 0.001 & 1.39 & $1.17-1.71$ \\
\hline & $\operatorname{ETS}\left(N^{*} / Y\right)$ & 0.05 & 0.5 & 1.06 & $0.88-1.27$ \\
\hline & Pet allergens $\left(N^{*} / Y\right)$ & -0.16 & 0.08 & 0.85 & $0.70-1.02$ \\
\hline \multirow{8}{*}{$\begin{array}{l}\text { Wheeze in } \\
\text { the last } \\
12 \text { months }\end{array}$} & $\operatorname{Sex}\left(F^{*} / M\right)$ & 0.22 & 0.08 & 1.25 & $0.97-1.61$ \\
\hline & Age groups: & & & & \\
\hline & $11-12 * / 13-15$ & -0.18 & 0.3 & 0.83 & $0.57-1.21$ \\
\hline & $6-10^{\star} / 11-12$ & -0.16 & 0.3 & 0.84 & $0.62-1.14$ \\
\hline & $\begin{array}{l}\text { Type of heating (heating with solid fuel*/ } \\
\text { central heating) }\end{array}$ & -0.37 & 0.02 & 0.68 & $0.49-0.94$ \\
\hline & Moulds/dampness $\left(\mathrm{N}^{*} / \mathrm{Y}\right)$ & 0.45 & 0.001 & 1.56 & $1.19-2.06$ \\
\hline & $\operatorname{ETS}\left(N^{*} / Y\right)$ & 0.01 & 0.9 & 1.01 & $0.83-1.22$ \\
\hline & Pet allergens $\left(N^{*} / Y\right)$ & -0.34 & 0.007 & 0.70 & $0.54-0.91$ \\
\hline
\end{tabular}


Table 6. Crude odds ratios (OR) and its 95\% confidence intervals related to determinants of observed health outcomes

\begin{tabular}{|c|c|c|c|c|c|}
\hline Health problem & $\begin{array}{l}\text { Determinants } \\
\text { *reference group }\end{array}$ & $\begin{array}{l}\text { Regression } \\
\text { coefficient }\end{array}$ & $\begin{array}{l}P \text {-value of the } \\
\text { regression coefficient }\end{array}$ & $\mathrm{OR}$ & $95 \% \mathrm{Cl}$ \\
\hline \multirow[t]{8}{*}{ Asthma } & $\operatorname{Sex}\left(F^{*} / M\right)$ & 0.4 & 0.001 & 1.54 & $1.19-1.99$ \\
\hline & Age groups: & & & & \\
\hline & $11-12 * / 13-15$ & 0.10 & 0.6 & 1.10 & $0.76-1.60$ \\
\hline & $6-10 * / 11-12$ & -0.25 & 0.1 & 0.77 & $0.56-1.06$ \\
\hline & $\begin{array}{l}\text { Type of heating (heating with solid fuel*/ } \\
\text { central heating) }\end{array}$ & -0.38 & 0.01 & 0.67 & $0.49-0.93$ \\
\hline & Moulds/dampness $\left(\mathrm{N}^{*} / \mathrm{Y}\right)$ & -0.3 & 0.06 & 0.72 & $0.52-1.0$ \\
\hline & ETS $\left(N^{*} / Y\right)$ & 0.1 & 0.2 & 1.17 & $0.90-1.52$ \\
\hline & Pet allergens $\left(N^{*} / Y\right)$ & -0.2 & 0.04 & 0.77 & $0.59-0.99$ \\
\hline \multirow[t]{8}{*}{ Bronchitis } & $\operatorname{Sex}\left(F^{\star} / M\right)$ & 0.25 & 0.01 & 1.28 & $1.05-1.57$ \\
\hline & Age groups: & & & & \\
\hline & $11-12 * / 13-15$ & 0.11 & 0.5 & 1.12 & $0.82-1.51$ \\
\hline & $6-10^{*} / 11-12$ & -0.48 & $<0.001$ & 0.61 & $0.48-0.79$ \\
\hline & $\begin{array}{l}\text { Type of heating (heating with solid fuel*/ } \\
\text { central heating) }\end{array}$ & -0.12 & 0.3 & 0.88 & $0.67-1.16$ \\
\hline & Moulds/dampness $\left(\mathrm{N}^{*} / \mathrm{Y}\right)$ & 0.36 & 0.001 & 1.44 & $1.15-1.80$ \\
\hline & $\operatorname{ETS}\left(N^{*} / \mathrm{Y}\right)$ & 0.15 & 0.1 & 1.17 & $0.95-1.43$ \\
\hline & Pet allergens $\left(N^{*} / Y\right)$ & -0.12 & 0.2 & 0.88 & $0.72-1.08$ \\
\hline \multirow{8}{*}{$\begin{array}{l}\text { Allergy to house } \\
\text { dust mite }\end{array}$} & $\operatorname{Sex}\left(F^{\star} / M\right)$ & 0.42 & $<0.001$ & 1.52 & $1.23-1.88$ \\
\hline & Age groups: & & & & \\
\hline & $11-12^{*} / 13-15$ & 0.14 & 0.3 & 1.15 & $0.86-1.54$ \\
\hline & $6-10 * / 11-12$ & -0.004 & 0.9 & 0.99 & $0.83-1.18$ \\
\hline & $\begin{array}{l}\text { Type of heating (heating with solid fuel }{ }^{*} / \\
\text { central heating) }\end{array}$ & -0.21 & 0.1 & 0.80 & $0.60-1.60$ \\
\hline & Moulds/dampness $\left(\mathrm{N}^{\star} / \mathrm{Y}\right)$ & 0.51 & $<0.001$ & 1.68 & $1.33-2.11$ \\
\hline & $\operatorname{ETS}\left(\mathrm{N}^{*} / \mathrm{Y}\right)$ & -0.02 & 0.8 & 0.97 & $0.77-1.24$ \\
\hline & Pet allergens $\left(\mathrm{N}^{*} / \mathrm{Y}\right)$ & -0.35 & 0.001 & 0.70 & $0.56-0.87$ \\
\hline \multirow[t]{8}{*}{ Allergy to pollen } & $\operatorname{Sex}\left(F^{*} / M\right)$ & 0.34 & $<0.001$ & 1.41 & $1.17-1.69$ \\
\hline & Age groups: & & & & \\
\hline & $11-12^{*} / 13-15$ & 0.08 & 0.5 & 1.09 & $0.85-1.39$ \\
\hline & $6-10 * / 11-12$ & 0.17 & 0.1 & 1.18 & $0.95-1.47$ \\
\hline & $\begin{array}{l}\text { Type of heating (heating with solid fuel }{ }^{*} / \\
\text { central heating) }\end{array}$ & 0.16 & 0.2 & 1.18 & $0.90-1.54$ \\
\hline & Moulds/dampness $\left(\mathrm{N}^{\star} / \mathrm{Y}\right)$ & 0.24 & 0.02 & 1.27 & $1.03-1.57$ \\
\hline & $\operatorname{ETS}\left(N^{*} / Y\right)$ & -0.08 & 0.3 & 0.92 & $0.76-1.11$ \\
\hline & Pet allergens $\left(N^{*} / Y\right)$ & -0.09 & 0.3 & 0.90 & $0.75-1.09$ \\
\hline \multirow[t]{8}{*}{ Pet allergy } & $\operatorname{Sex}\left(F^{*} / M\right)$ & 0.30 & 0.01 & 1.36 & $1.05-1.75$ \\
\hline & Age groups: & & & & \\
\hline & $11-12^{*} / 13-15$ & 0.05 & 0.8 & 1.05 & $0.72-1.51$ \\
\hline & $6-10 * / 11-12$ & -0.03 & 0.8 & 0.96 & $0.70-1.31$ \\
\hline & $\begin{array}{l}\text { Type of heating (heating with solid fuel*/ } \\
\text { central heating) }\end{array}$ & -0.09 & 0.5 & 0.90 & $0.64-1.28$ \\
\hline & Moulds/dampness $\left(\mathrm{N}^{*} / \mathrm{Y}\right)$ & 0.35 & 0.01 & 1.42 & $1.07-1.89$ \\
\hline & $\operatorname{ETS}\left(\mathrm{N}^{*} / \mathrm{Y}\right)$ & 0.04 & 0.7 & 1.04 & $0.79-1.04$ \\
\hline & Pet allergens $\left(N^{*} / Y\right)$ & -0.52 & $<0.001$ & 0.59 & $0.45-0.76$ \\
\hline
\end{tabular}


The hygiene hypothesis offers some insight into the relationship between exposure to indoor environmental factors and the development of allergic diseases and asthma. It proposes that the rising prevalence of these diseases may be linked to such factors as: increased sanitary regime in dwellings, bad dietary habits, urbanization, and the adoption of Western lifestyles. Reduced microbial stimulation can lead to a Th1/Th2 lymphocyte imbalance. Consequently, the Th2 phenotype dominant in foetal life is fixed, which may in turn lead to IgE hypersensitivity to existing allergens $[13,14]$. Currently, the hygiene hypothesis has been complemented with the gut microbial deprivation hypothesis, indicating the protective role of microorganisms in the development of allergy and asthma. Excessive sanitation of the environment contributes to a reduction in the number of infections, changes the intestinal colonization by reducing exposure to microorganisms and, by disrupting the development of the immune system, is a predisposing factor for the development of allergies and asthma. A large number of studies confirm the assumptions of that hypothesis, showing that certain internal allergens, microorganisms present in the home environment with which a child comes into contact from an early age, may reduce the risk of allergies, and asthma [6]. The Urban Environment and Childhood Asthma (URECA) study conducted in a cohort of children from American cities in populations with a high prevalence of asthma showed that high exposure to cockroach, mouse, and cat allergens in infancy was a protective factor for the development of asthma at 7 years of age [15]. Moreover, interesting findings emerge from studies in a group of American agricultural populations leading a traditional lifestyle: the Amish and the Hutterites [16]. A significant difference between those groups lies in their attitude to modern technologies: the former use traditional agricultural practices, whereas the latter make use of advanced solutions in their work. The findings of studies conducted in those communities point to a much lower prevalence of asthma among the Amish. Moreover, the dust in their dwellings showed a higher concentration of endotoxins, which would confirm the microbial deprivation hypothesis.

The findings of our own research indicate the protective effect of the presence of pets on the prevalence of asthma, the occurrence of allergies to pet allergens, allergy to house dust mite, and wheeze in the last 12 months. The exposure of children to pet allergens continues to be a valid and controversial research subject. The results of the conducted analyses do not provide a clear answer as to the protective or causal influence of that exposure. Similarly, recommendations regarding bronchial asthma in childhood do not give clear guidelines as to the exposure to allergens of that type. As regards allergic diseases, allergen immunotherapy (AIT) is a well-known and effective method of treating them, although such treatment has not thus far been used for bronchial asthma.
In 2019, the European Academy of Allergy and Clinical Immunology devised guidelines on the application of house dust mites (HDM) AIT as an add on treatment for HDM driven allergic asthma [17]. That therapy has its limitations: it is recommended for controlled or partly controlled allergic asthma. The use of pet allergens, pollen, and moulds in AIT in asthma requires further research. A diagnosis of allergy or bronchial asthma may therefore force a family to take a difficult decision whether to keep their pet or get rid of it.

The results of cross-sectional studies do not address sufficiently the direction of the influence of exposure to pet allergens on allergic diseases, respiratory symptoms, bronchitis, and asthma. Their practical impact should thus be analysed with great caution on a caseby-case basis. Some authors have also suggested that the protective effect of exposure to pets may only be apparent and may in fact result from selective avoidance of exposure by patients or high-risk individuals [18-20]. Neither can the effect of reverse causation be ruled out in the authors' own research, given that the information obtained by means of the questionnaire is limited. This is the more so since the data from the study indicate statistically significant differences between families with asthma keeping a pet (56.2\%) and those without such a history (62.9\%). Similarly, a diagnosed mould allergy has a statistically significant influence on keeping a pet. Regardless of the limitations of the cross-sectional study, the results of the regression analysis give a clear picture and add a practical dimension to the problem not only in relation to the recommendations for children with asthma or allergic diseases, but also as regards the methodological questions of the conducted studies. For the sake of completeness, it is necessary to acquire additional information on the contact with a pet and/or the moment at which it is avoided.

The results of own research confirm that moulds present in children's dwellings are a risk factor for asthma, respiratory symptoms, bronchitis, and allergic diseases under study. This observation is confirmed by other studies. It is indicated that the presence of moulds in dwelling increases the risk of developing wheeze by $53 \%$ and asthma by $56 \%[7,8]$. Increased humidity stimulates the growth of fungi and some bacteria that can trigger inflammatory processes in the respiratory tract. Furthermore, it was shown that in the case of asthma, fungal sensitization may increase the severity of the disease and carry a higher risk of hospitalization [21].

Heating homes with solid fuel was a risk factor for asthma and wheezing in the past 12 months. It should be noted that the study distinguished only between central heating and heating with solid fuel. The latter form of heating included the use of coal, wood, pellets, or ecopea coal. The questions used in the questionnaire made it impossible to differentiate between the various types of solid fuel used for heating dwellings and conduct the 
relevant analyses. It is possible that such differentiation would produce results different from those presented above. Particular studies provide epidemiological evidence of a link (or lack of it) between the type of fuel used and the risk of developing allergic diseases and asthma. A recent meta-analysis found that indoor exposure to wood burning is not associated with an increased risk of asthma [22]. The studies aimed at assessing the relationship between heating of the dwellings by means of gas and the prevalence of asthma do not yield clear answers as to the direction of that influence. It is only emphasized that exposure to $\mathrm{NO}_{2}$ may also come from other sources and their effect is difficult to estimate [23].

The conducted study did not confirm the importance of ETS for the prevalence of the diseases and symptoms under investigation. Such a relationship was observed only in the case of chronic cough. These remarks should also be interpreted through the prism of the limitations of the cross-sectional study, in particular limited information on exposure. The mere fact of smoking tobacco did not mean that it occurred in the presence of a child. The questionnaire did not contain data on the place of smoking (inside or outside the dwelling) and it seems difficult to estimate the time and intensity of the exposure. As regards the observed relationship with the prevalence of chronic cough, it must be recalled that it may be one of the predictors of bronchial asthma. Moreover, studies point to health and socioeconomic burdens associated with that symptom [24]. The proven links between ETS and other diseases should promote the reduction of children's exposure as far as possible.

Other limitations of the study should be also indicated. They result from the applied study model (crosssectional) and used definitions. First of all, there are some predictive limitations of the study. For example, it remains unclear whether the home environment can be treated as constants. It was defined based on questionnaire data and probably it is related to the place of the child's residence during the study. We have not collected any information about the history of residence. Therefore, it cannot be excluded that the presence of respiratory symptoms and diseases may be related to the previous place of residence. The study result could also be influenced by the group size. The response rate was $59.5 \%$, but taking into consideration the minimum sample size, it corresponds to theoretical and methodological assumptions.

\section{Conclusions}

The study confirmed the adverse influence of the presence of moulds, heating with solid fuel on the prevalence of asthma, bronchitis, respiratory symptoms, and allergic diseases. The protective influence of pets on the occurrence of the health disorders under study was dem- onstrated, yet given the type of study used, the results must be interpreted with great caution.

\section{Conflict of interest}

The authors declare no conflict of interest.

\section{References}

1. Pawankar R, Canonica GW, Holgate ST, Lockey RF (eds). The WAO White Book of Allergy 2011. World Allergy Organization. United Kingdom 2011.

2. Mallol J, Cranol J, von Mutis E, et al. The International Study of Asthma and Allergies in Childhood (ISAAC) Phase Three: A global synthesis. Allergol Immunopathol 2013; 41: 73-85.

3. Samoliński B, Raciborski F, Łaskawiec A, et al. Epidemiologia Chorób Alergicznych w Polsce (ECAP). Alergol Pol 2014; 1: 10-8.

4. Wypych-Ślusarska A, Czech E, Kasznia-Kocot J, et al. Health inequalities among students of lower secondary school in Bytom, Poland. Ann Agric Environ Med 2018; 25: 4-8.

5. del Giudice M, Allegorico A, Parisi G, et al. Risk factors for asthma. Ital J Pediatr 2014; 40 (Suppl 1): A77.

6. Fuchs O, Genuneit J, Latzin P, et al. Farming environments and childhood atopy, wheeze, lung function, and exhaled nitric oxide. J Allergy Clin Immunol 2012; 130: 382-8 e6.

7. Fisk WJ, Lei-Gomez Q, Mendell MJ. Meta-analyses of the associations of respiratory health effects with dampness and mold in homes. Indoor Air 2007; 17: 284-96.

8. Castro-Rodriguez JA, Forno E, Rodriguez-Martinez CE, Celedón JC. Risk and protective factors for childhood asthma: what is the evidence? J Allergy Clin Immunol Pract 2016; 4: 1111-22.

9. Kim BJ, Lee SY, Kim HB, et al. Environmental changes, microbiota and allergic diseases. Allergy Asthma Immunol Res 2014; 6: 389-400.

10. West C, Jenmalm M, Prescott S. The gut microbiota and its role in the development of allergic disease: a wider perspective. Clin Exp Allergy 2015; 45: 43-53.

11. Wilmore W, Aldrige K. Infectious asthma triggers: time to revise the hygiene hypothesis? Trends Microbiol 2015; 23: 389-91.

12. Główny Urząd Statystyczny. Ludność. Stan i struktura oraz ruch naturalny w przekroju terytorialnym w 2017 r. Stan w dniu 31 XII. https://stat.gov.pl/obszary-tematyczne/ludnosc/ludnosc/ludnosc-stan-i-struktura-oraz-ruch-naturalnyw-przekroju-terytorialnym-w-2017-r-stan-w-dniu-31-xii,6,23. html (accessed on 27.12.2020).

13. Brooks C, Pearce N, Douwes J. The hygiene hypothesis in allergy and asthma: an update. Curr Opin Immunol 2013; 13: 70-7.

14. Braun-Fahrländer C. Mikrobielles Umfeld und allergische Erkrankungen im Kindesalter. Ther Umsch 2013; 70: 714-9.

15. O'Connor G, Lynch SV, Bloomnerg GE, et al. Early-life home environment and the risk of developing asthma among inner-city children. J Allergy Clin Immunol 2018; 141: 1468-75.

16. Stein MM, Hrusch CL, Gozdz J, et al. Innate immunity and asthma risk in Amish and Hutterite Farm children. N Engl J Med 2016; 375: 411-21.

17. Agache I, Laculiceanu A, Cojanu C, et al. Advances in allergen immunotherapy for asthma. Curr Opin Allergy Clin Immunol 2020 Aug 6. doi: 10.1097/ACI.0000000000000686. 
18. AlShatti KA, Ziyab AH. Pet-Keeping in relation to asthma, rhinitis, and eczema symptoms among adolescents in Kuwait: a cross-sectional study. Front Pediatr 2020; 8: 331.

19. Rutter CE, Silverwood RJ, Williams HC, et al. Are environmental factors for atopic eczema in ISAAC phase three due to reverse causation? J Invest Dermatol 2019; 139: 1023-36.

20. Silverwood RJ, Rutter CE, Mitchell EA, et al. Are environmental risk factors for current wheeze in the International Study of Asthma and Allergies in Childhood (ISAAC) phase three due to reverse causation? Clin Exp Allergy 2019; 49: 430-41.

21. Kao CC, Hanania NA, Parulekar AD. The impact of fungal allergic sensitization on asthma. Curr Opin Pulm Med 2021; 27: 3-8.

22. Guercio V, Pojum IC, Leonardi GS, et al. Exposure to indoor and outdoor air pollution from solid fuel combustion and respiratory outcomes in children in developed countries: a systematic review and meta-analysis. Sci Total Environ 2020; 755: 142187.

23. Heinrich J. Influence of indoor factors in dwellings on the development of childhood asthma. Int J Hyg Environ Health 2011; 214: 1-25.

24. Dicpingaitis P. Understanding the Foundations of Chronic Cough. Am J Manag Care 2020; 26 URL: https://www.ajmc. $\mathrm{com} / \mathrm{view} /$ understanding-the-foundations-of-chroniccough. 